No. 4189 February 11, 1950

The disappearance of periodate is followed in all cases by the appearance of free iodine; carbon dioxide can be detected, and the insoluble oxidized cotton has an appreciable carboxyl content not to be accounted for on the basis of the accepted mechanism of oxidation.

Similar results have been obtained for the oxidation of $\beta$-methyl glucoside and $\beta$-methyl cellobioside with sodium metaperiodate. With a large excess of oxidant, the reaction does not terminate when the consumption of periodate and production of formic acid have reached the limits set by the classical Malaprade reaction, unless the experiment is carried out in complete darkness. In bright daylight, oxidation proceeds more rapidly and goes very much further.

There are very great variations in the rates of oxidation of different $\alpha$-glycols (carbohydrates, glycosides, etc.) by periodate. It is probable that the disturbance of the normal reaction caused by irradiation is most pronounced when the 'dark reaction' is relatively slow, as it is in the oxidation of cellulose.

It is worthy of note that solutions of sodium metaperiodate are themselves unstable when kept in bright daylight (for example, the concentration of a $0.03 M$ solution fell by about 10 per cent during fifteen days), and the odour of ozone first observed by Rammelsberg 4 is very pronounced. Solutions kept in the dark are both odourless and stable ; illuminated reaction mixtures of sodium metaperiodate with cotton or the glycosides do not smell of ozone.

Such evidence as is yet available points to the occurrence of far-reaching degradation during the photochemical reaction. The investigation of the subject is being continued in these laboratories.

Frank S. H. Head

British Cotton Industry Research Association, Shirley Institute,

Didsbury, Manchester. Sept. 20.

1. Text. Inst, 38, T389 (1947),

'J. Amer. Chem. Soc., 59, 2049 (1937).

${ }^{3} J$. Text. Inst., 32, T109 (1941).

4 Pogg. Ann., 134, 534 (1868).

\section{Isolation of a Saponin from the Heart-wood of the Mora Tree and of a Related Species, Morabukea}

IN the course of a chemical examination of the wood of the mora tree, Mora excelsa (Benth.), from British Guiana and from Trinidad, we observed that the heart-wood contains an unusually high proportion of material soluble in water or alcohol, of which up to about one-half was found to consist of a saponin.

The saponin is most readily isolated by exhaustive extraction of the wood with hot alcohol, evaporation of the alcoholic liquor nearly to dryness, and trituration of the dark-coloured syrup with an excess of hot acetone. The colouring matter and other impurities pass into solution in the acetone, leaving a light-coloured amorphous solid, which is separated and purified by further treatment with acetone. The saponin is finally obtained as a very pale buff- or pink-coloured powder, the aqueous solution of which exhibits intense foaming properties. The saponin content of the heart-wood of a number of samples of mora from British Guiana was found to lie between 2.8 and 9.6 per cent, based on the dry weight of the wood. A saponin has also been found in the heart. wood of mora from Trinidad, and of the related species morabukea (Mora gonggrijpii (Kleinh.) Sandwith); but these woods have not been examined quantitatively. Although saponins are known to occur in small quantities in the wood of the boles of a few tree species ${ }^{1}$, the wood of mora may prove to be a relatively rich source.

On hydrolysis with 6 per cent alcoholic hydrochloric acid for $30 \mathrm{~min}$. at the boiling point, mora saponin yielded 21.4 per cent of a crude crystalline aglycone. Fractional crystallization of this product from ethyl alcohol resulted in the isolation, first, of a crystalline sapogenin of melting point $269-270^{\circ}$ and $[\alpha]_{D}+29 \cdot 5^{\circ}(c=0.83$ in chloroform $)$ in a yield of $\mathbf{9 . 4}$ per cent of the dry weight of the original saponin (found : C, 76.67; H, 10.63 per cent). This sapogenin readily forms a monoacetate of melting point $255-257^{\circ}$ (found: C, 76.91; H, 10.14 per cent). At least one other crystalline sapogenin is known to be present in the hydrolysis product of the original saponin. These substances are being further examined by Dr. D. H. R. Barton, to whom we are indebted for the suggestion that the principal sapogenin of melting point $269-270^{\circ}$ is a new triterpenoid hydroxycarboxylic acid.

Hydrolysis of mora saponin with 5 per cent aqueous sulphuric acid at $95^{\circ}$ for $4 \mathrm{hr}$. gave a mixture of sugars from which the aglycone was separated by filtration. After neutralization of the filtrate with barium carbonate and evaporation to dryness, the sugars were extracted with 90 per cent ethyl alcohol and isolated as a concentrated syrup. Examination of the syrup by partition chromatography on paper ${ }^{2}$ revealed the presence of two sugars, which were probably glucose and xylose.

The research upon which this communication is based was carried out as part of the programme of the Forest Products Research Board on behalf of the Colonial Products Research Council. It is published by permission of the Department of Scientific and Industrial Research and the Colonial Products Research Council.

$$
\begin{aligned}
& \text { R. H. FARMER } \\
& \text { W. G. CAMPBELI }
\end{aligned}
$$

Section of Wood Chemistry,

Forest Products Research Laboratory, Princes Risborough, Aylesbury, Bucks.

$$
\text { Oct. } 3 .
$$
'Wise, L. E., et al., "Wood Ohemistry", 436 (Reinhold, New York, Partridge, S. M., Nature, 158, 270 (1946).

\section{Preparation of Carbonate-free Sodium Hydroxide}

WE have been studying the carbonate - hydroxide ion exchange on the strong base resin 'Amberlite IRA-400' (manufactured by Rohm and Haas Com. pany and supplied by Messrs. Charles Lennig and Co., Ltd., 1-11 John Adam Street, London, W.C.2). It was expected that the bivalent ion would be preferentially held by the resin, and this is so. The following figures show the results of bringing the resin in the carbonate form into equilibrium with various sodium hydroxide solutions. 